\title{
Synthesis and characterization of a novel Y-Fe phase via kinetic neutron diffraction
}

Editorial Enterprises Marked Proof $\mathrm{CM} / 121483 / \mathrm{PAP}$ 22941ap

Printed on $01 / 05 / 01$ at 16.10

\author{
Susan H Kilcoyne ${ }^{1,3}$, Pascal Manuel ${ }^{1}$ and Clemens Ritter ${ }^{2}$ \\ ${ }^{1}$ Department of Physics and Astronomy, University of Leeds, Leeds, LS2 9JT, UK \\ 2 Institut Laue Langevin, 6 rue Jules Horowitz, BP156, 38042, Grenoble Cedex 9, France \\ E-mail: s.h.kilcoyne@leeds.ac.uk
}

Received 29 January 2001, in final form 22 March 2001

\begin{abstract}
Kinetic in situ neutron diffraction has been used to study the crystallization of amorphous $\mathrm{Y}_{67} \mathrm{Fe}_{33}$. The results show that partial crystallization first occurs close to $300^{\circ} \mathrm{C}$ where the $\mathrm{Y}$ phase is formed. The entire sample crystallizes at $390^{\circ} \mathrm{C}$ and new Bragg peaks appear, signifying the formation of a novel $\mathrm{Y}-\mathrm{Fe}$ phase. This new phase coexists with $\mathrm{Y}$ to $450^{\circ} \mathrm{C}$ when the Bragg peaks associated with this phase rapidly decrease in intensity and $\mathrm{YFe}_{2}$ also coexisting with Y, emerges as the final crystallization product. Rietveld refinement shows that the new phase crystallizes into a hexagonal structure, space group $P 6_{3} / m m c$, with $a=12.8893(7) \AA, c=11.7006(9) \AA$ and $\gamma=120^{\circ}$.
\end{abstract}

(Some figures in this article are in colour only in the electronic version; see www.iop.org)

\section{Introduction}

The structural phase diagrams of most binary rare earth-transition metal (RE-TM) systems have been studied in great detail and it is generally considered that most parent phases have been identified and classified, and only minor modifications associated with perhaps interstitial substitution or site selectivity remain to be characterized. Indeed, no entirely new binary RE-TM structural phases have been identified for some years. However, the majority of equilibrium phase diagrams have been determined under conditions that are far from equilibrium. Generally, binary mixes are annealed at pre-determined temperatures and subsequently quenched to ambient temperatures and examined. This method has a severe limitation: the assumption that the phases and phase proportions present at the annealing temperature are retained after quenching has occurred must always be made. Here we show how high intensity kinetic neutron diffraction can be used to circumvent this limitation by allowing an in situ exploration of the phase diagrams of binary alloys. The phase diagrams can therefore be mapped in detail by studying phase formations and transformations at the temperatures at which they occur.

${ }^{3}$ Correspondence should be addressed to Dr S H Kilcoyne, Department of Physics and Astronomy, University of Leeds, Leeds, LS2 9JT, UK. 


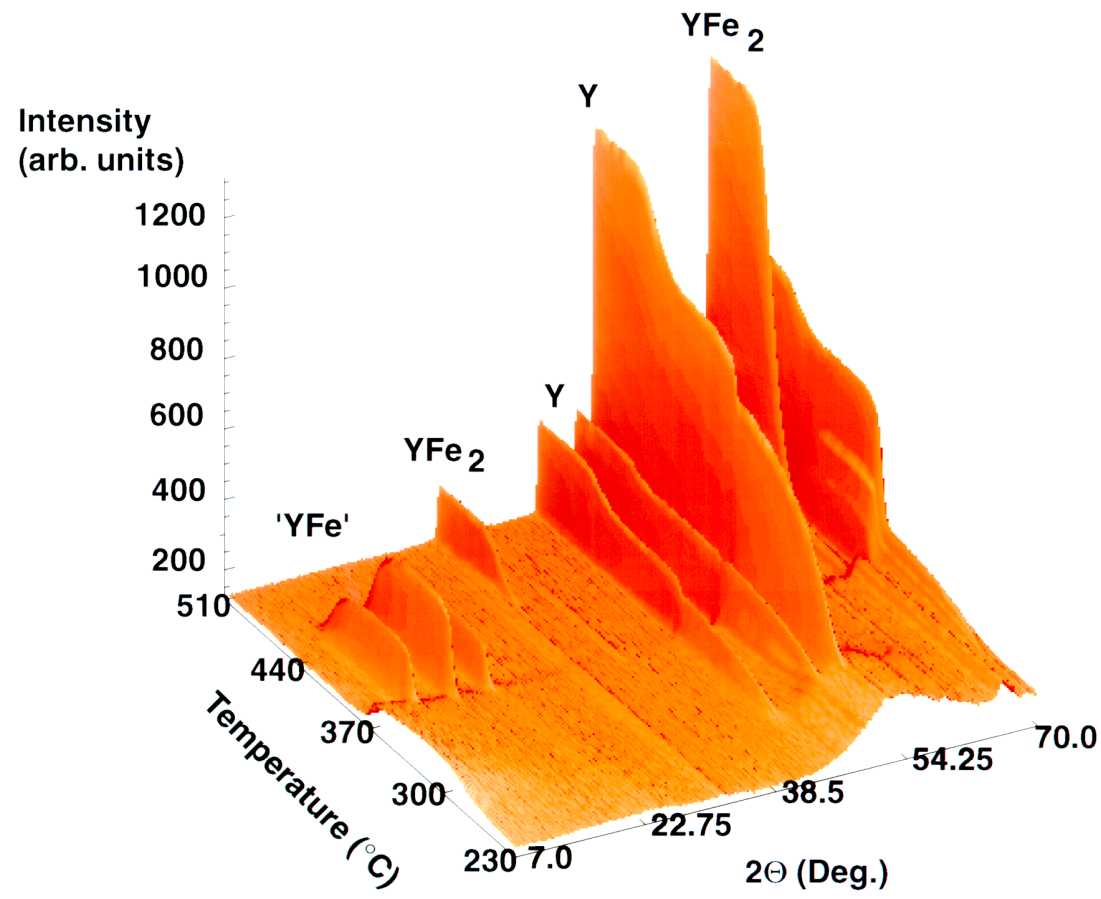

Figure 1. Neutron thermogram of crystallization of $\mathrm{Y}_{67} \mathrm{Fe}_{33}$ on ramping the temperature from $230^{\circ} \mathrm{C}$ to $510^{\circ} \mathrm{C}$. Note the presence of the intermediate $\mathrm{Y}-\mathrm{Fe}$ phase between $400^{\circ} \mathrm{C}$ and $450^{\circ} \mathrm{C}$. The final phase is $\mathrm{Y}$ and $\mathrm{YFe}_{2}$.

As part of a wider investigation of binary RE-TM phase diagrams using neutron diffraction techniques we have adopted the novel procedure of annealing amorphous RE-Fe precursors in the neutron beam and monitoring the resulting phase formations and transformations via neutron thermograms. This method, which has previously proved effective in the preparation of high quality superconducting cuprates and nanocystalline alloys (see e.g. [1-3] and references therein) has led to the identification of a new intermetallic compound with a novel structure and unusual magnetic and electronic properties.

Studies of the crystallization of melt-spun amorphous $\mathrm{Y}_{1-x} \mathrm{Fe}_{x}$ alloys were carried out by Tenhover in 1981 [4] and by Croat in 1982 [5]. Tenhover studied the crystallization of splat-cooled amorphous $\mathrm{Y}_{0.66} \mathrm{Fe}_{0.34}$ using ${ }^{57} \mathrm{Fe}$ Mössbauer spectroscopy, differential scanning calorimetry (DSC), $\mathrm{x}$-ray diffraction and high temperature resistivity. His results indicated that the crystallization occurs in two stages: firstly the formation of $\mathrm{Y}$, followed by the formation of $\mathrm{YFe}_{2}$. He saw no evidence of any other phases. Croat studied the crystallization processes in a range of melt spun amorphous $\mathrm{Y}_{1-x} \mathrm{Fe}_{x}$ alloys with $0.4 \leqslant x \leqslant 0.79$ using DSC and $\mathrm{x}$-ray diffraction. The DSC measurements indicated that while the Fe rich alloys with $0.6 \leqslant x \leqslant 0.79$ crystallize directly into the appropriate metallic phase, the Y rich alloys with $x=0.4,0.45$ and 0.5 exhibit a multi-stage crystallization process with the formation of an intermediate metastable phase. Croat investigated this phase in more detail by stopping the DSC at the appropriate temperatures, cooling the sample to room temperature and collecting an $\mathrm{x}$-ray diffraction pattern from the quenched material. While diffraction patterns collected from partially crystallized $\mathrm{Y}_{0.6} \mathrm{Fe}_{0.4}$ quenched from $600 \mathrm{~K}$ showed Bragg peaks arising solely from $\mathrm{Y}$, and patterns collected from material quenched from $750 \mathrm{~K}$ showed reflections due to $\mathrm{Y}$ and 
$\mathrm{YFe}_{2}$, the X-ray pattern of partially crystallized $\mathrm{Y}_{0.6} \mathrm{Fe}_{0.4}$ quenched from $\sim 450 \mathrm{~K}$ contained additional Bragg peaks, which he attributed to an unidentifiable metastable Y-Fe phase. Similar results were also obtained for samples with $x=0.45$ and 0.50 . Croat made no other studies of this phase or its formation and did not attempt to determine its crystal structure.

In this paper we present the results of an in situ crystallization of high purity, amorphous $\mathrm{Y}_{67} \mathrm{Fe}_{33}$ melt-spun ribbons using kinetic neutron diffraction. Using this technique we have synthesized a novel Y-Fe compound and, via Rietveld refinement, have determined its structure.

\section{Experimental procedure}

Amorphous ribbons of $\mathrm{Y}_{67} \mathrm{Fe}_{33}$ were prepared by an $\mathrm{RF}$ melt spinning technique in which molten $\mathrm{Y}_{67} \mathrm{Fe}_{33}$ is ejected at high velocity onto a rapidly rotating copper wheel, and quenched at $\sim 10^{6} \mathrm{~K} \mathrm{~s}^{-1}$. $3 \mathrm{~g}$ of the resulting amorphous ribbon were sealed in a vanadium can and loaded into a furnace mounted on the high flux D20 powder diffractometer at the Institut Laue Langevin (ILL). Neutron diffraction patterns were collected over the angular range $2 \theta=5^{\circ}$ to $135^{\circ}$ every four minutes while the furnace temperature was ramped smoothly from $230^{\circ} \mathrm{C}$ to $510^{\circ} \mathrm{C}$ at a rate of $40^{\circ} \mathrm{C}$ per hour. The incident neutron wavelength was $2.4 \AA$. In addition to the spectra collected on D20, a high resolution diffraction pattern was collected from a sample prepared by stopping the crystallization process at $375^{\circ} \mathrm{C}$ and cooling rapidly to room temperature. This spectrum was collected at ambient temperature using the high resolution diffractometer D2B at the ILL with $\lambda=1.594 \AA$ over the angular range $2 \theta=6^{\circ}$ to $140^{\circ}$.

\section{Results and discussion}

The neutron thermogram collected on D20 is shown in figure 1, where it can be seen that partial crystallization of $\mathrm{Y}_{67} \mathrm{Fe}_{33}$ first occurs at approximately $300^{\circ} \mathrm{C}$. At this temperature a pure $\mathrm{HCP}$ elemental $\mathrm{Y}$ phase is formed, with the Fe atoms segregating to form an Fe rich amorphous phase. There is also a marked increase in the scattering at low angles as the Y phase forms. At $390{ }^{\circ} \mathrm{C}$ there is a dramatic crystallization of the entire sample, witnessed by an abrupt loss of the amorphous contribution to the diffraction pattern. At the same temperature new Bragg peaks appear, signifying the formation of an intermediate phase which coexists with the $\mathrm{Y}$ matrix to $\sim 450{ }^{\circ} \mathrm{C}$. At this temperature, the Bragg peaks associated with this intermediate phase rapidly decrease in intensity, there is a concomitant further increase in the intensity of the $\mathrm{Y}$ peaks, and the well known cubic $\mathrm{C} 15$ Laves phase $\mathrm{YFe}_{2}$ emerges. The final crystallization product is $\mathrm{YFe}_{2}$ coexisting in equilibrium with crystalline elemental $\mathrm{Y}$. The temperature dependence of the initial crystallization, the formation of $\mathrm{Y}$ and the formation and transformation of the Y-Fe phases are particularly apparent when the low angle $\left(5^{\circ}<2 \theta<50^{\circ}\right)$ region of the thermogram is viewed from the side, as shown in figure 2.

In the following sections we shall examine the nature of each of these phases in more detail.

\subsection{Low angle scattering}

The increase in intensity of the scattering at low angles, $2^{\circ}<2 \theta<25^{\circ}$, is seen clearly in figures 1 and 2 . The scattering first appears at $\sim 275^{\circ} \mathrm{C}$, and rapidly increases in intensity reaching a peak at $310^{\circ} \mathrm{C}$. Above this temperature, the intensity of the scattering decreases slowly until at $375{ }^{\circ} \mathrm{C}$ the whole sample crystallizes and the scattering disappears. The scattering appears and starts to increase rapidly at the temperature at which Y first starts to 


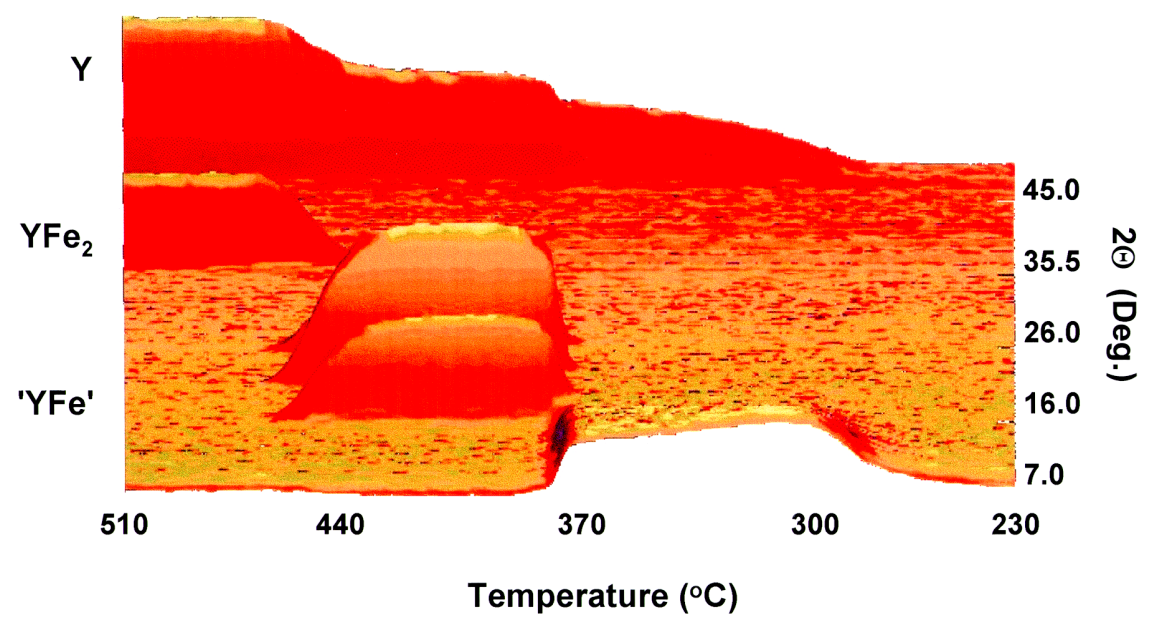

Figure 2. The low angle $\left(5^{\circ}<2 \theta<50^{\circ}\right)$ section of the neutron thermogram viewed from the side. Diffraction patterns collected at low temperatures are shown on the right-hand side of the figure, and spectra collected at higher temperature are shown to the left.

form, and it disappears when the entire sample crystallizes. It would seem reasonable therefore to suggest that this scattering is due to microcrystallites of $\mathrm{Y}$ in the amorphous matrix.

\subsection{Formation of crystalline $Y$}

The first Bragg peaks to be observed in the neutron thermogram arise from elemental Y. Rietveld refinement using FullProf [6] showed this to be a pure HCP Y phase, space group $P 6_{3} / \mathrm{mmc}^{\mathrm{m}}$ with $a=3.650(1) \AA, c=5.77(1) \AA, \alpha=\beta=90^{\circ}$ and $\gamma=120^{\circ}$ at $327^{\circ} \mathrm{C}$. Figure 3(a) shows the intensity of the $\mathrm{Y}(100)$ Bragg peak as a function of temperature, where four distinct regions are apparent. Below $300{ }^{\circ} \mathrm{C}$ no crystallization has taken place, and the intensity at the position of the $\mathrm{Y}(100)$ reflection is due to scattering from the amorphous material. Y Bragg peaks first begin to appear at approximately $300{ }^{\circ} \mathrm{C}$, after which there is a steady rise in the intensity of the Bragg peak as the temperature increases to $375^{\circ} \mathrm{C}$. Between 375 and $390{ }^{\circ} \mathrm{C}$ the intensity of the Y Bragg peaks increases dramatically and there is a sudden crystallization of the sample, accompanied by the appearance of the intermediate Y-Fe phase. This phase exists until $\sim 450^{\circ} \mathrm{C}$ when there is a third and final increase in intensity of the $\mathrm{Y}(100)$ as the $\mathrm{YFe}_{2}$ phase is formed in coexistence with the pure $\mathrm{Y}$ phase.

As might be expected, the Y Bragg peaks sharpen considerably during the annealing process, with a dramatic decrease in the $\mathrm{FWHH}$ occurring at $\sim 375^{\circ} \mathrm{C}$, the temperature at which the whole sample crystallizes. The FWHH of the Bragg peaks provide information on the dimensions of the Y grains via the Scherrer formula [7]

$$
t=\frac{0.9 \lambda}{B \cos \theta} \text {. }
$$

In this expression $t$ is particle size in $\AA, \lambda$ is the neutron wavelength ( $2.4 \AA$ in this experiment), $\theta$ is half the scattering angle and $B$ is the FWHH of a Bragg peak in radians. $B$ is related to the FWHH of a Bragg peak from the sample, $B_{S}$, and the FWHH of a Bragg peak from an yttrium-iron-garnet (YIG) standard, $B_{Y I G}$, by the expression $B^{2}=B_{S}^{2}-B_{Y I G}^{2}$. The size of the $\mathrm{Y}$ grains determined from these equations is shown as a function of temperature in figure 3(b). The evolution of the Y phase can be seen quite clearly. Y grains of $\sim 170 \AA$ appear at $\sim 310^{\circ} \mathrm{C}$, 


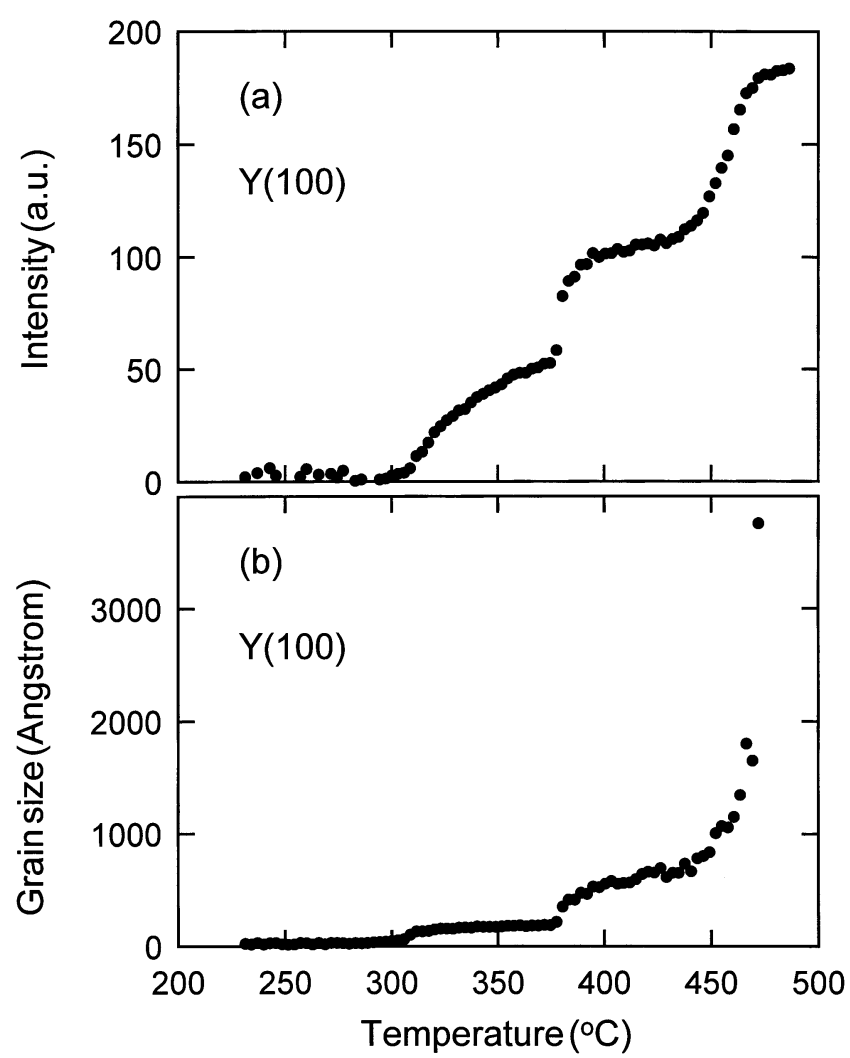

Figure 3. (a) Temperature dependence of the normalized intensities of the Y (100) Bragg peak, (b) size of Y grains determined from the (100) peak width as described in the text.

and remain to $\sim 350{ }^{\circ} \mathrm{C}$. Above $350{ }^{\circ} \mathrm{C}$ these grains grow steadily, reaching $\sim 800 \AA$ at $450{ }^{\circ} \mathrm{C}$. At higher temperatures, grain growth is extremely rapid and by $\sim 475^{\circ} \mathrm{C}$ the determination of the grain size is limited by the resolution of the instrument.

\subsection{The intermediate phase}

At $390{ }^{\circ} \mathrm{C}$ Bragg peaks, which are not part of the Y pattern, appear and then disappear at $450{ }^{\circ} \mathrm{C}$ when the $\mathrm{YFe}_{2}$ phase is formed. As the composition of the precursor material is known it is possible to determine the chemical composition of the intermediate phase from the intensity of the pure $\mathrm{Y}$ peaks and the intensity of the $\mathrm{Y}$ and $\mathrm{YFe}_{2}$ peaks in the final phase, and we have identified the composition of this intermediate phase as close to stoichiometric YFe, a phase that is entirely absent from all published phase diagrams of the Y-Fe system, although we believe it to be related to the phase observed by Croat in [5].

The absence of this intermediate phase from the phase diagrams is perhaps not surprising: it can be seen in figure 4 that the phase is truly stable only over a temperature range of $40{ }^{\circ} \mathrm{C}$. Attempts to produce crystalline $\mathrm{YFe}$ by argon arc melting $\mathrm{Y}$ and $\mathrm{Fe}$ have proved unsuccessful, and clearly the formation of the YFe phase requires the intimate atomic mixing of constituents afforded by the amorphous precursor in order to form. However, we have found that it is possible, by rapidly cooling the sample from $375^{\circ} \mathrm{C}$, to stabilize this phase at room temperature, where it coexists in equilibrium with pure Y. It should be noted that 


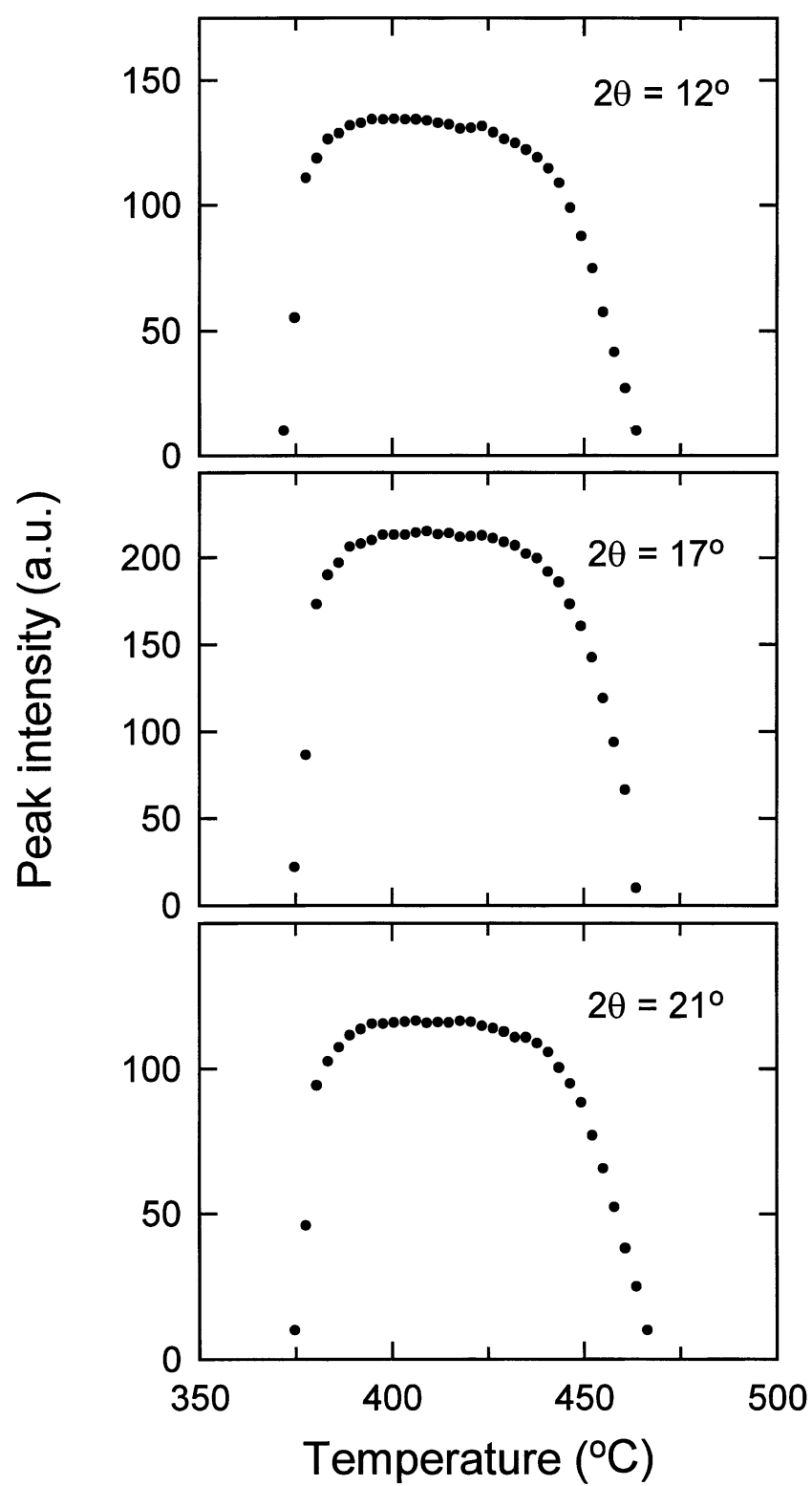

Figure 4. Temperature dependence of the peak intensity of the (100), (110) and (210) reflections arising from the intermediate $\mathrm{Y}-\mathrm{Fe}$ phase.

the crystallization and subsequent rapid cooling was carried out in the neutron beam and we are certain from the diffraction patterns collected during the cooling process that the phases existing at room temperatures are the same as those observed at high temperature.

Figure 5 shows the high resolution neutron diffraction pattern collected on D2B at ambient temperature from a sample of crystalline $\mathrm{Y}$ and $\mathrm{Y}-\mathrm{Fe}$. A comparison of the diffraction pattern collected at $420^{\circ} \mathrm{C}$ on D20 and the pattern collected on D2B confirms that the phase has not transformed during the quenching process and we are confident that any structural information 


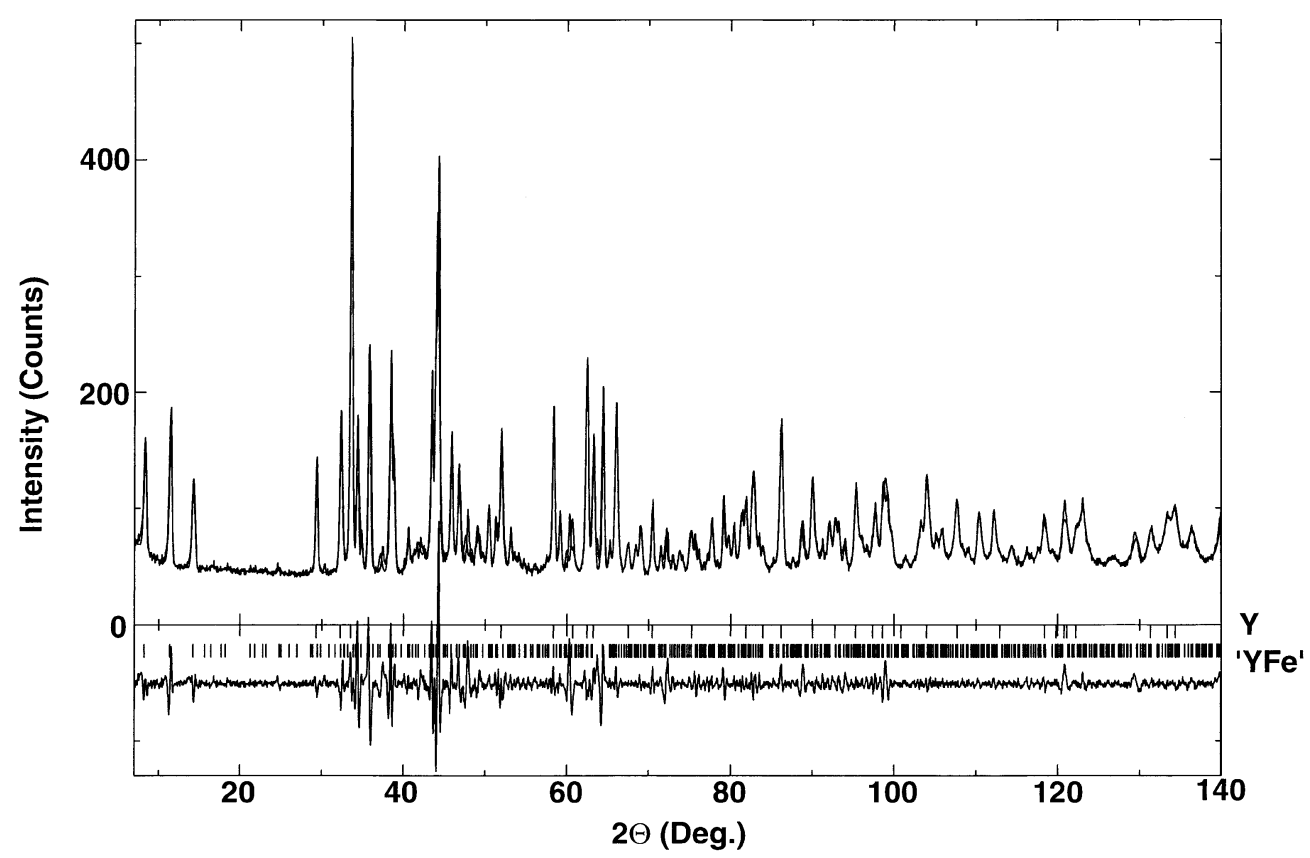

Figure 5. Observed, calculated and difference powder neutron diffraction pattern of the new YFe phase within an Y matrix.

obtained from our room temperature, high resolution data relates to the phase which exists at high temperatures. The solid line in figure 5 shows the data and the result of a two-phase Rietveld refinement using FullProf [6]. A description of the YFe phase is obtained using the pattern matching option and the crystal structure of the $\mathrm{Y}$ phase is obtained from a full Rietveld refinement of the spectrum. From this ab initio structural method we have established that the YFe phase crystallizes with the hexagonal space group $P 6_{3} / m m c$ with $\gamma=120^{\circ}$ and unusually large cell dimensions of $a=12.8893(7) \AA$ and $c=11.7006(9) \AA$, giving a cell volume of 1683.781(1) $\AA^{3}$. The best fit of the Y spectrum is found using a hexagonal cell, space group $P 6_{3} / m m c$, with $a=3.6417(2) \AA$ and $c=5.7383(5) \AA$, in good agreement with the reported values of $a=3.647 \AA$ and $c=5.728 \AA$ [8]. The details of the refinement are given in table 1. As yet, we have not determined the exact positions of the $\mathrm{Y}$ and $\mathrm{Fe}$ atoms in this structure, although it is clear that a cell of this volume will contain a large number of atoms. By examination of the Fe-Fe and Y-Fe distances in other $\mathrm{Y}-\mathrm{Fe}$ phases $(\sim 2.6 \AA$ and $\sim 3.5 \AA$ respectively) and assuming a density of $7 \mathrm{~g} \mathrm{~cm}^{-3}$ we propose a structure containing 90-100 atoms/unit cell.

By applying the Scherrer equation (equation (1)) to the Y-Fe reflections between $12^{\circ}$ and $22^{\circ}$ we have determined that, when the Y-Fe phase crystallizes, grains of $250 \pm 11 \AA$ are formed immediately. These grains do not grow as the temperature increases, but remain this size until $460{ }^{\circ} \mathrm{C}$, when the phase disappears.

\subsection{Formation of $\mathrm{YFe}_{2}$}

At $\sim 445^{\circ} \mathrm{C}$ the Bragg peaks associated with the intermediate phase begin to decrease in intensity, and peaks associated with the final crystallization product, the $\mathrm{C} 15$ Laves phase $\mathrm{YFe}_{2}$, begin to appear. This phase co-exists with elemental $\mathrm{Y}$ at all temperatures. The diffraction 
Table 1. Results of a two-phase refinement of the diffraction pattern shown in figure 5. The parameters for phase $1(\mathrm{Y})$ were determined using a full Rietveld refinement of the data. Those for phase 2 (YFe) were determined using the profile matching option in FullProf.

\begin{tabular}{ll}
\hline $\begin{array}{l}\text { Phase } 1 \mathrm{Y} \\
\text { (Rietveld refinement) }\end{array}$ & $\begin{array}{l}\text { Phase } 2 \text { Y-Fe } \\
\text { (Profile matching) }\end{array}$ \\
\hline $\begin{array}{l}\text { Space group } P 6_{3} / m m c \\
a=3.6417(2) \AA\end{array}$ & $\begin{array}{l}\text { Space group } P 6_{3} / m m c \\
a=12.8903(7) \AA\end{array}$ \\
$c=5.7383(5) \AA$ & $c=11.7015(9) \AA$ \\
$\alpha=\beta=90^{\circ} \quad \gamma=120^{\circ}$ & $\alpha=\beta=90^{\circ} \quad \gamma=120^{\circ}$ \\
$V=65.9037(6) \AA^{3}$ & $V=1683.781(1) \AA^{3}$ \\
Bragg $R$-factor $=3.95$ & Bragg $R$-factor $=0.291$ \\
$R_{f}$-factor $=2.03$ & $R_{f}$-factor $=0.264$ \\
\hline
\end{tabular}

Table 2. Results of a two-phase refinement of the diffraction pattern shown in figure 6 . The parameters for both phases were determined using a full Rietveld refinement of the data.

\begin{tabular}{|c|c|}
\hline Phase $1 \mathrm{Y}$ & Phase $2 \mathrm{YFe}_{2}$ \\
\hline Space group $P 6_{3} / m m c$ & Space group $F d 3 m$ \\
\hline$a=3.659(1) \AA$ & $a=7.416(3) \AA$ \\
\hline$c=5.791(4) \AA$ & \\
\hline$\alpha=\beta=90^{\circ}$ & $\alpha=\beta=\gamma=90^{\circ}$ \\
\hline$V=67.14(4) \AA^{3}$ & $V=407.858(2) \AA^{3}$ \\
\hline Bragg $R$-factor $=2.50$ & Bragg $R$-factor $=3.99$ \\
\hline$R_{f}$-factor $=2.37$ & $R_{f}$-factor $=2.32$ \\
\hline
\end{tabular}

pattern obtained from the final material at $490^{\circ} \mathrm{C}$ is shown in figure 6 , and, with the exception of a small peak at $2 \theta \sim 59^{\circ}$, no Bragg peaks other than those associated with either $\mathrm{Y}$ or $\mathrm{YFe}_{2}$ are observed. The best fit is obtained with a two-phase Rietveld refinement with one phase of elemental Y, space group $P 6_{3} / m m c$ with $a=3.659(1) \AA$ and $c=5.791(4) \AA$, and a second phase of cubic $\mathrm{YFe}_{2}$, space group $F d \overline{3} m$ with $a=7.416(3) \AA$. The results of the Rietveld refinement are shown as a solid line in figure 6 and the accompanying parameters are listed in table 2.

In addition to this structural study we have also examined the magnetic properties of this new Y-Fe phase using low temperature neutron diffraction, ${ }^{57} \mathrm{Fe}$ Mössbauer spectroscopy, muon spin relaxation and dc magnetization techniques. Our measurements have revealed that the new Y-Fe compound is a weak itinerant electron antiferromagnet with a Néel temperature close to $55 \mathrm{~K}$ and a magnetic moment of less than $1 \mu_{B}$ [9].

We have also extended our studies of phase formation in Y-Fe to phase formation in other RE-TM systems. In addition to the new YFe phase obtained from the crystallization of amorphous $\mathrm{Y}_{67} \mathrm{Fe}_{33}$ ribbons, we have found evidence of similar new and exotic binary magnetic and crystallographic phases in systems such as Er-Fe and $\mathrm{Y}-\mathrm{Co}$. As an example consider the Er-Fe system: crystallization of $\alpha-\mathrm{Er}_{67} \mathrm{Fe}_{33}$ proceeds via the initial crystallization of pure $\mathrm{Er}$ and as the temperature increases Bragg peaks associated with the C15 Laves phase $\mathrm{ErFe}_{2}$ emerge. The final crystallization product is $\mathrm{ErFe}_{2}$ coexisting in equilibrium with crystalline Er. However, careful examination of the diffraction patterns collected between $350{ }^{\circ} \mathrm{C}$ and $390{ }^{\circ} \mathrm{C}$ show the presence of weak Bragg peaks at $2 \theta=12,17$ and $22^{\circ}$, indicating that, like $\mathrm{Y}-\mathrm{Fe}$, crystallization occurs via the formation of an intermediate, stoichiometric RE-Fe phase. 


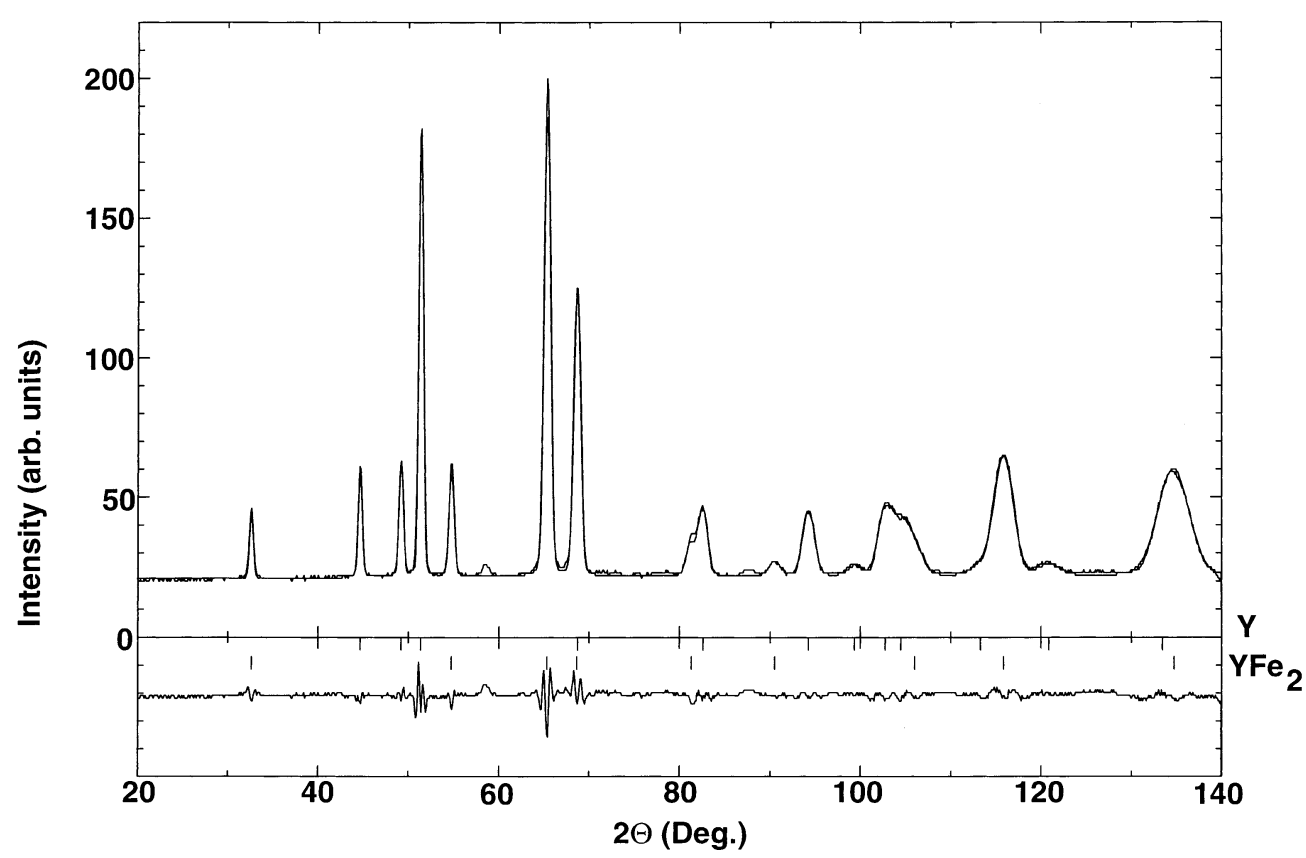

Figure 6. Observed, calculated and difference powder neutron diffraction pattern of the final phase: $\mathrm{YFe}_{2}$ within an $\mathrm{Y}$ matrix.

\section{Conclusions}

This method of studying phase formation is clearly very powerful and provides information on phase formation not obtained by other methods. It is envisaged that the results of this work will form a valuable input into theoretical ab initio augmented spherical wave (ASW) [10] and selfconsistent spin-polarized linear muffin-tin orbital (LMTO) [11] band structure calculations of the interplay of structural, electronic and magnetic properties in precisely the families of intermetallic compounds upon which our investigations are based. In many cases the agreement between the predictions of ASW and LMTO calculations and existing experimental data are extremely good. However, the parameter space available to the theoreticians is often restricted by the lack of appropriate stable inter-metallic compounds against which the calculations can be tested. Through kinetic neutron diffraction techniques we are already synthesizing novel and relevant crystallographic phases, which will undoubtedly stimulate further calculations and provide a stringent test of the predictive capabilities of these methods.

\section{Acknowledgments}

The authors are grateful to Drs Pierre Convert and Thomas Hansen for their help during the D20 experiment and to Professor Bob Cywinski for his invaluable comments. The UK EPSRC and French MENESR (PM) are acknowledged for their financial support.

\section{References}

[1] Boardman C J, Kilcoyne S H and Cywinski R 1991 Recrystallization of $\alpha-\mathrm{Bi}_{2} \mathrm{Sr}_{2} \mathrm{CaCu}_{2} \mathrm{O}_{x}$ studied using small angle neutron scattering Physica C 185-189 633-4 
[2] Boardman C J, Kilcoyne S H and Cywinski R 1992 Kinetic neutron studies of the recrystallization of amorphous $\mathrm{Bi}-\mathrm{Sr}-\mathrm{Ca}-\mathrm{Cu}-\mathrm{O}$ Physica B 180-181 426-8

[3] Fernandez Barquin L, Gomez Sal J C, Gorria P, Garitaonandia J S and Barandiaran J M 1998 Crystal structure and magnetic behaviour of nanocrystalline $\mathrm{Fe}-\mathrm{Nb}-\mathrm{Cu}-\mathrm{Si}-\mathrm{B}$ alloys studied by means of in-situ neutron diffraction J. Phys.: Condens. Matter 10 5027-38

[4] Tenhover M 1981 A study of the crystallization process in the metallic glass $\mathrm{Y}_{0.66} \mathrm{Fe}_{0.34}$ J. Phys. F: Met. Phys. 11 2697-706

[5] Croat J J 1982 Melt-spun yttrium-iron alloys: magnetic properties and crystallization kinetics J. Appl. Phys. 53 6932-40

[6] Rodriguez-Carvajal J 1993 Recent advances in magnetic structure determination by neutron powder diffraction Physica B 192 55-69

[7] Cullity B D 1956 Elements of X-ray Diffraction (Reading MA: Addison-Wesley)

[8] 1992 Metals and Alloys Index JCPDS-ICDD

[9] Kilcoyne S H, Manuel P, Hillier A D and Ritter C The magnetic properties of a new Y-Fe phase in preparation

[10] Coehoorn R 1989 Calculated electronic structure and magnetic properties of Y-Fe compounds Phys. Rev. B 39 $13072-85$

[11] Sabiryanov R F and Jaswal S S 1998 Electronic structure and magnetic properties of Y-Fe compounds Phys. Rev. B 57 7767-72 
Annotations from 22941ap.pdf

\section{Page 1}

Annotation 1

Please be aware that any colour figures in this article will only be printed in colour if you have previously arranged this with the Production Editor

\section{Page 8}

Annotation 1

' $R \_f$ ' as intended in tables 1 and 2 ?

\section{Page 10}

Annotation 1

Please update reference [9] 